\title{
Estimación de $Q$ utilizando la transformada robusta de Fourier en registros Check-shot VSP.
}

Rómulo Sandoval Flórez, Universidad de los Andes, Universidad de Pamplona.

José Luís Paredes, Universidad de los Andes.

Flor Alba Vivas Mejía, Instituto Colombiano del petróleo/Ecopetrol.

Copyright 2016, SBGf - Sociedade Brasileira de Geofísica

Este texto foi preparado para a apresentação no VII Simpósio Brasileiro de Geofísica, Ouro Preto, 25 a 27 de outubro de 2016. Seu conteúdo foi revisado pelo Comitê Técnico do VII SimBGf, mas não necessariamente representa a opinião da SBGf ou de seus associados. É proibida a reprodução total ou parcial deste material para propósitos comerciais sem prévia autorização da SBGf.

\section{Resumen}

En este trabajo se usa la transformada robusta de Fourier en el cálculo del factor de calidad $Q$ en registros verticales de pozo (VSP). Los métodos de corrimiento del centroide de la frecuencia y frecuencia de pico son severamente afectados por la presencia de ruido aditivo no gaussiano. El método es probado en trazas sintéticas contaminadas con ruido impulsivo, donde se compara el valor calculado de $Q$ utilizando la transformada de Fourier y la transformada robusta. Este trabajo ha sido financiado por la Universidad de Pamplona, Colciencias y Ecopetrol.

\section{Introducción}

Los registros verticales de pozo check shot, son usados para calibrar la velocidad del pozo, en ellos se coloca la fuente cercana a la vertical del pozo y se graban los disparos en geófonos ubicados en una herramienta dentro del pozo. El ruido en los datos proviene de cuatro fuentes (Galperin 1985), el ruido ambiente generado en superficie, las ondas de tubo, hydro-ondas y las ondas de cable.

Generalmente el ruido impulsivo tiene gran amplitud y no puede ser modelado como ruido gaussiano. Este tipo de ruido puede ser clasificado como datos erráticos (Claerbout and Muir, 1973) o como datos contaminados con ruido errático (Trickett et al., 2012). En el campo de la estadística robusta, los datos erráticos son conocidos como outliers (Maronna et al., 2006).

Los métodos robustos han sido utilizados en el procesamiento de datos sísmicos para la supresión de ruido (Chen, 2013); Guitton la usa en la inversión robusta usando la transformada de Huber (Guitton and Symes, 2003) y Liu en el proceso de deconvolución para remoción de reflexiones multiples (Liu and Lu, 2014).

En este trabajo se presentan los resultados obtenidos utilizando los métodos robustos para el cálculo de $Q$ en datos sintéticos VSP contaminados con ruido impulsivo.

Métodos para el cálculo de $\mathbf{Q}$

\section{Método del corrimiento del centroide de la frecuencia}

Es conocido que en el fenómeno de dispersión la atenuación aumenta con la frecuencia, las frecuencias altas son más rápidamente atenuadas que las bajas, lo que hace que el centroide de la frecuencia se desplace hacia las frecuencias más bajas y es posible cuantificar el valor de $Q$ a partir de este corrimiento. Este método fue desarrollado por Quan y Harrist (1997), quienes definen el corrimiento del centroide de la frecuencia (CFS) por la expresión

$f_{c}=\frac{\int_{0}^{\infty} f S(f) d f}{\int_{0}^{\infty} S(f) d f}$

donde $S(f)$ es el espectro de Fourier de la onda $s(t)$, asumiendo que el espectro de la fuente tiende una forma gaussiana. Para el cálculo de $Q$ se utiliza la expresión

$Q=-\frac{\pi \Delta t \sigma^{2}}{f_{s}-f_{c}}$

donde $f_{c}$ es la frecuencia de pico del receptor, $f_{S}$ es la frecuencia de pico de la fuente, $\Delta t$ es el tiempo de las dos interfaces y $\sigma$ es la varianza de la fuente que está determinada por

$\sigma^{2}=\frac{\int_{0}^{\infty}\left(f_{c}-f_{s}\right)^{2} s(f) d f}{\int_{0}^{\infty} s(f) d f}$

\section{Método de la frecuencia de pico}

El método de la frecuencia de pico (PF) fue propuesto por Zhang y Ulrych (2002) para estimar el factor $Q$ en datos CMP. Se considera el espectro de la fuente como tipo Ricker y se deduce la expresión para el cálculo de $Q$ como

$Q=\frac{\pi \Delta t f_{p} f_{p 0}^{2}}{2\left(f_{p 0}^{2}-f_{p}^{2}\right)}$,

donde $f_{p 0}$ es la frecuencia de pico de la fuente y $f_{p}$ es la frecuencia de pico después de un tiempo $\Delta t$, la relación de la frecuencia del centroide y la frecuencia de pico es

$f_{p}=\frac{\sqrt{\pi}}{2} f_{c}$

Donde $f_{p}$ es la frecuencia de pico y $f_{p}$ es la frecuencia del centroide

\section{Modelamiento de la traza sintética:}

Para el modelamiento de las trazas sísmicas sintéticas se utilizó el siguiente procedimiento: Se considera una onda plana en un medio viscoelástico, expresada en la forma:

$P(z, \omega)=P_{0}(\omega) e^{i[k(\omega) z-\omega t]}$, 
donde $P_{0}(\omega)$ es la transformada de Fourier del pulso de la fuente $y$

$k(\omega)=\frac{\omega}{c(\omega)}=\frac{\omega}{c_{p}(\omega)}+i \alpha(\omega)$,

es el número de onda complejo.

Para modelar la atenuación y la dispersión se utiliza el modelo convolucional de una señal propagándose en un medio disipativo:

$u(\omega)=r(\omega) w(\omega) e^{-i k(\omega) \Delta z}$,

donde $r(\omega)$ es la reflectividad (posición del geófono), $w(\omega)$ es la transformada de la fuente y $k(\omega)$ es la atenuación

$k(\omega)=\frac{\omega}{c}\left(\frac{\omega^{\gamma}}{\omega_{r} \gamma}\right)^{-1} e^{-i\left(\frac{1-\gamma}{2 Q}\right)}$

donde $\omega_{r}$ es la frecuencia de referencia, $c$ es la velocidad de propagación, $Q$ es el factor de calidad y $\gamma=\frac{1}{2 Q}$.

El sismograma mostrado en la Figura 1, se generó para modelar un experimento donde los geófonos están ubicados a una distancia de $300 \mathrm{~m}$ y $600 \mathrm{~m}$ en profundidad, la velocidad de propagación de la onda es de $1000 \mathrm{~m} / \mathrm{s}$ y la frecuencia de referencia es de $\omega_{r}=$ $30 \mathrm{~Hz}$._La propagación se da en un medio homogéneo con factor de atenuación $Q=25$. Se observa que las frecuencias altas se atenúan más rápidamente y el centroide de la frecuencia se corre hacia atrás.
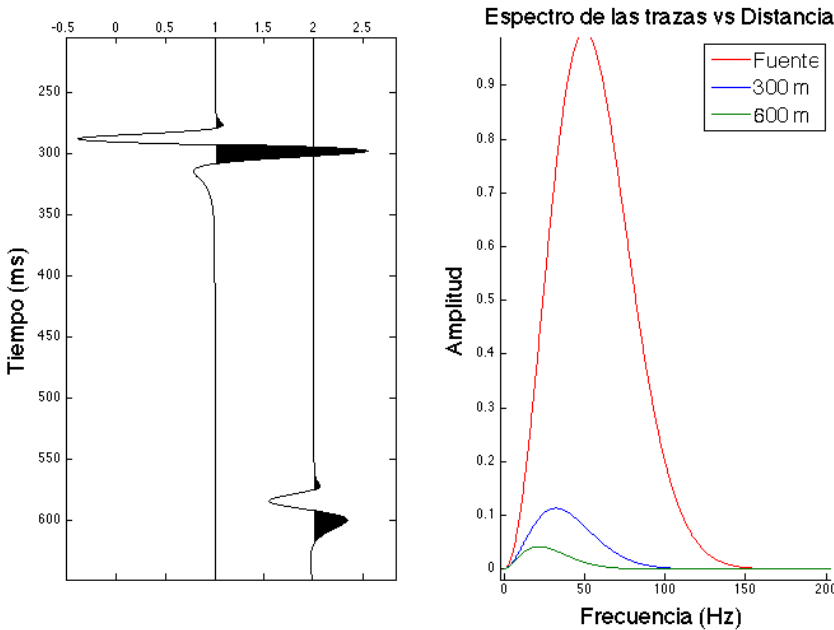

Figura 1. Izquierda: traza sintética. Derecha: Espectro de frecuencia para la fuente y la señal en los dos receptores.

\section{Ruido impulsivo}

Pruebas realizadas con_datos reales de registros Check shot VSP, utilizando la determinación de la norma $p$ de la distribución gaussiana generalizada, nos indican que el ruido es Laplaciano, es por ello que se escoge modelar el ruido como de colas pesadas.
Las trazas sísmicas son contaminadas con ruido impulsivo modelado como "e-contaminated". Una metodología del modelamiento de procesos nogaussianos, empieza del modelo gaussiano y es modificado adicionando muestras inapropiadas. La función de densidad del "e-contaminated" toma la siguiente forma:

$f(x)=(1-\epsilon) f_{n}(x)+\epsilon f_{c}(x)$

donde $f_{n}(x)$ es la densidad nominal gaussiana con varianza $\sigma_{n}^{2}, \epsilon$ es una constante que determina el porcentaje de contaminación y $f_{c}(x)$ es la contaminación gaussiana de gran varianza, tal que $\sigma_{c}^{2}=10 \sigma_{n}^{2}$.

\section{Metodología}

\section{Transformada Robusta}

Una transformada permite representar una señal en tiempo discreto en un dominio alternativo donde la señal de entrada es mejorada para su posterior análisis. Una transformación unitaria puede ser definida como $X=\phi z$, donde $z=\left[z_{1}, z_{2}, \ldots, z_{n}\right]^{T}$ denota la señal en tiempo discreto, $x=\left[x_{1}, x_{2}, \ldots x_{n}\right]^{T}$ es la señal mapeada, $\phi \in \mathbb{C}^{N \times N}$, para una transformada unitaria $z=\phi^{p} X$.

Para obtener los coeficientes de la transformada que mejor describe la señal de interés desde las muestras con ruido impulsivo, el algoritmo robusto es desarrollado bajo las estimación MAP( Maximum a posteriori). Se asume que las perturbaciones adicionan ruido laplaciano (Paredes, 2015). Tomando el ajuste a la norma $l_{1}$, por lo tanto, la transformada se reduce a resolver el problema de regresión usando LAD Mínima desviación absoluta. Esto es

$X=\operatorname{argmin}\left\{|| y-\Psi X||_{l_{1}}+\lambda|| X||_{l_{1}}\right\}$

En la Figura 2, se presentan los resultados de la transformada robusta y la transformada convencional de Fourier de la Wavelet Ricker de $50 \mathrm{~Hz}$ contaminada con ruido impulsivo. El cálculo de la frecuencia de pico mediante las ecuaciones (1) y (5), usando la transformada de Fourier y la transformada Robusta de Fourier, muestra que mejores resultados son obtenidos con la transformada robusta.

\section{Resultados}

Dado que el objetivo del presente trabajo es valorar la transformada robusta como técnica para estimación de la frecuencia pico y la posterior estimación de $Q$, se realizaron experimentos numéricos para estimar el valor del factor de calidad $Q$ en trazas sintéticas.

Las trazas sintéticas fueron contaminadas con un porcentaje de ruido de $5 \%$ y $10 \%$ respectivamente, la relación señal ruido varia de 5 a $30 \mathrm{~dB}$ y los parámetros usados para el modelamiento fueron: frecuencia de la fuente de $50 \mathrm{~Hz}, Q=50, z=300$ y $600 \mathrm{~m}$ y $\omega_{r}=30 \mathrm{~Hz}$. 
El valor de $Q$ es calculado para un número de 30 realizaciones para cada valor de relación señal ruido.
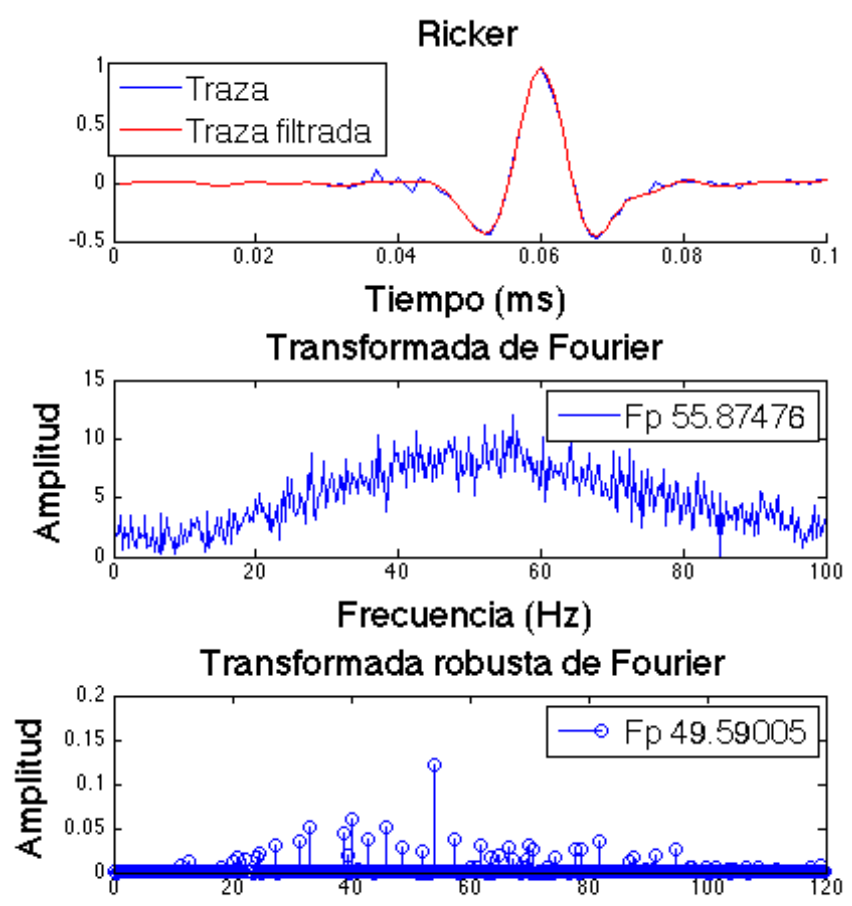

Frecuencia $(\mathrm{Hz})$

Figura 2. a) Señal contaminada con ruido impulsivo (azul) y señal filtrada usando la transformada robusta de Fourier (rojo) b) Frecuencia de pico estimada usando la transformada de Fourier c) Frecuencia de pico estimada usando la transformada robusta de Fourier.

\section{Cálculo de $\mathrm{Q}$}

En la Figura 3 se muestran los valores de $Q$ calculados para las trazas contaminadas con un porcentaje de $10 \%$ de impulsividad.

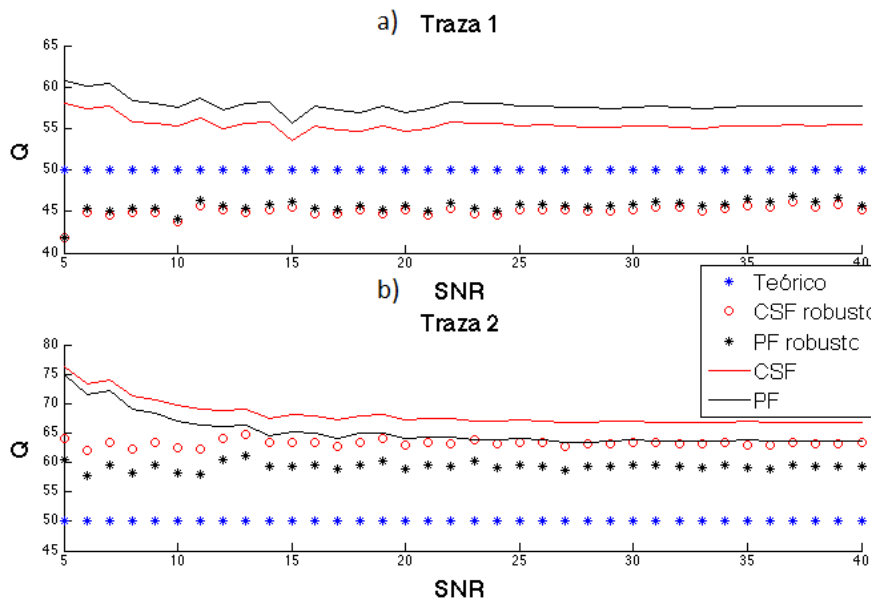

Figura 3. Valor estimado de $Q$ vs. Relación señal ruido (SNR) para un porcentaje de contaminación del 10\%
En la Figura 4 se muestran los valores de $Q$ calculados para las trazas contaminadas con un porcentaje de $5 \%$ de impulsividad.

a) Traza 1

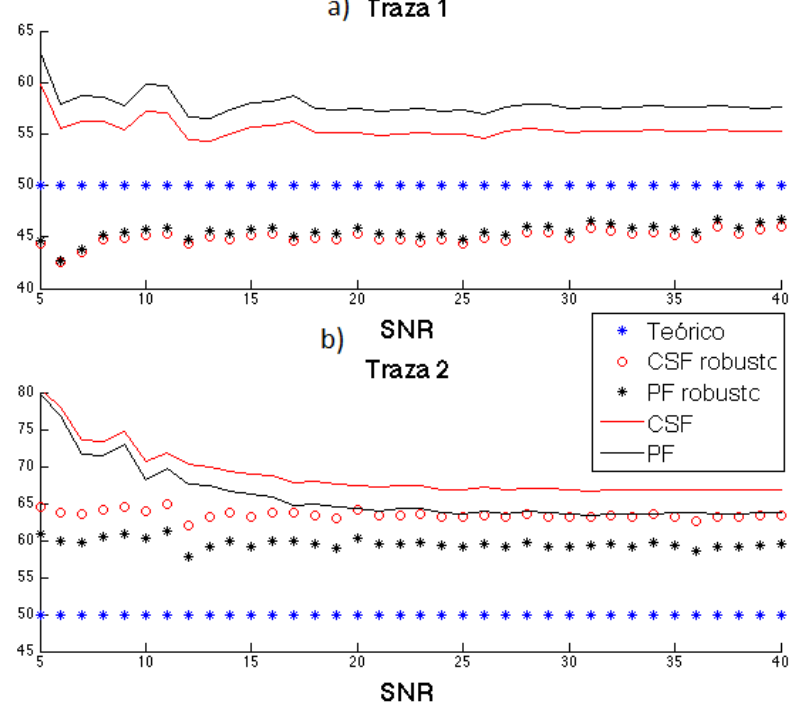

Figura 4. Valor estimado de $Q$ vs. Relación señal ruido (SNR) para un porcentaje de contaminación del 5\%

\section{Discusión de resultados}

1. El valor de $Q$ calculado por el método de frecuencia de pico se acerca más al valor teórico de 50.

2. Al aumentar la profundidad el valor calculado de $Q$ se aleja del valor teórico, lo cual se debe a que aumenta la asimetría en la ondicula por la dispersión.

3. El método es robusto en cuanto al porcentaje de contaminación.

4. El cálculo de $Q$ mejora con la transformada robusta al comparar los resultados usando la transformada de Fourier.

\section{Agradecimientos}

Rómulo Sandoval agradece su beca doctoral al proyecto 531 financiado por Colciencias, Ecopetrol y la Universidad de Pamplona.

\section{Bibliografía}

Chen, Ke. 2013. "Robust matrix rank reduction methods for seismic data processing" Tesis de Maestria, Universidad de Alberta.

Claerbout, Jon F. y Francis Muir. 1973. "Robust modelling with erratic data". Geophysics 38:826-844.

Galrperin, E. I. 1885. "Borehole seismology".D. reidel publishing company.

Guiton, Antonie y William Symes. 2003. "Robust inversion of seismic data using the Huber norm" Geophysics 684:1310-1319.

Liu, Lei, y Wenkai Lu. 2014. "Non-Gaussianity based Time Varying Predictive Deconvolution for Multiple 
Removal."SEG.Technical Program Expanded Abstracts 2014.

Maronna, Ricardo A., R. D. Martin, y Victor Yohai. 2006. Robust Statistics. Editado por David J. Balding et all. John Wiley \& Sons.

Quan, Youli, y Jerry M. Harrist. 1997. "Seismic attenuation tomography using the frequency shift method." Geophysics 62 (3): 895-905.

Ramírez, Juan Marcos, and José Luis Paredes. 2015. "Robust Transforms Based on the Weighted Median Operator." IEEE signal processing letters 22-1:120-124.

Trickett, Stewart, Lynn Burroughs, y Andrew Milton. 2012. "Robust Rank reduction filtering for erratic noise." SEG Las Vegas 2012 Annual Meeting.

Zhang, Changjun, y Tadeusz J. Ulrych. 2002. "Estimation of quality factors from CMP records." Geophysics 67 (5): 1542-1547. 